International Journal of Pure and Applied Mathematics

Volume 93 No. 5 2014, 699-714

ISSN: 1311-8080 (printed version); ISSN: 1314-3395 (on-line version)

url: http://www.ijpam.eu

doi: http://dx.doi.org/10.12732/ijpam.v93i5.9

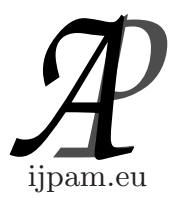

\title{
NON COMMUTATIVE FOURIER TRANSFORM AND \\ PLANCHEREL THEOREM FOR THE AFFINE GROUP
}

\author{
Kahar El-Hussein ${ }^{1 \S}$, Badahi Ould Mohamed $^{2}$ \\ ${ }^{1}$ Department of Mathematics \\ Faculty of Science \\ Al Furat University \\ Dear El Zore, SYRIA \\ ${ }^{1,2}$ Department of Mathematics \\ Faculty of Arts Science at Al Qurayat \\ Al-Jouf University, KINGDOM OF SAUDI ARABIA
}

\begin{abstract}
Let $G=S L(2, \mathbb{R})$ be the $2 \times 2$ connected real semisimple Lie group. Let $A F=\mathbb{R}^{2} \rtimes G L(2, \mathbb{R})$ be the affine group, which is the semidirect product of the two groups $\mathbb{R}^{2}$ with $G L(2, \mathbb{R})$, whivh plays an important role in technology. The purpose of this paper is to define the Fourier transform in order to obtain the Plancherel formula for the group $S L(2, \mathbb{R})$, and then we establish the Plancherel theorem for the group $P=\mathbb{R}^{2} \rtimes S L(2, \mathbb{R})$. To this end a Plancherel theorem for the affine group $A F=\mathbb{R}^{2} \rtimes G L(2, \mathbb{R})$ will be obtained
\end{abstract}

AMS Subject Classification: 43A30, 35D05

Key Words: Iwasawa decomposition, affine group $\mathbb{R}^{2} \rtimes G L(2, \mathbb{R})$, Fourier transform, Plancherel theorem

\section{Introduction}

1. Abstract harmonic analysis is the field in which results from Fourier anal-

Received: March 18, 2014

(C) 2014 Academic Publications, Ltd.

${ }^{\S}$ Correspondence author url: www.acadpubl.eu 
ysis are extended to topological groups which are not commutative that has connections to, theoretical physics, chemistry analysis, algebra, geometry, and the theory of algorithms. The classical Fourier transform is one of the most widely used mathematical tools in engineering. However, few engineers know that extensions Fourier analysis on noncommutative Lie groups holds great potential for solving problems in robotics, image analysis, mechanics. Engineering applications of noncommutative harmonic analysis brings this powerful tool to the engineering world. The Fourier transform, known in classical analysis, and generalized in abstract harmonic analysis. For a long time, people have tried to construct objects in order to generalize Fourier transform and Pontryagin, $\mathrm{s}$ theorem to the non abelian case. However, with the dual object not being a group, it is not possible to define the Fourier transform and the inverse Fourier transform between $G$ and $\widehat{G}$. These difficulties of Fourier analysis on noncommutative groups makes the noncommutative version of the problem very challenging. It was necessary to find a subgroup or at least a subset of locally compact groups which were not "pathological", or "wild" as Kirillov calls them [12]. Unfortunately if the group $G$ is no longer assumed to be abelian, it is not possible anymore to consider the dual group $\widehat{G}$. (i.e the set of all equivalence classes of unitary irreducible representations). Abstract harmonic analysis on locally compact groups is generally a difficult task. Still now neither the theory of quantum groups nor the representations theory have done to reach this goal. Recently, these problems found a satisfactory solution with the papers $[4,5,8,11]$. The ways were introduced in those papers will be the business of the expertise in the theory of abstract harmonic analysis, and in theoretical physics, and that is what I am interested. In this paper I will define the Fourier transform onthe group $A F^{+}=\mathbb{R}^{2} \rtimes G L_{+}(2, \mathbb{R})$ in order to obtain the Plancherel theorem on the affine group $A F \simeq \mathbb{R}^{2} \rtimes G L(2, \mathbb{R})$. where

$$
G L_{+}(2, \mathbb{R})=\left\{\left(X=\begin{array}{ll}
a & b \\
c & d
\end{array}\right): \operatorname{det} X>0\right\}
$$

\section{Fourier Transform and Plancherel Formula on Space Time $S L(2, \mathbb{R})$}

2. In the following and far away from the representations theory of Lie groups we use the Iwasawa decomposition of $S L(2, \mathbb{R})$, to define the Fourier transform and to demonstrate Plancherel formula on the connected real semisimple Lie group $S L(2, \mathbb{R})$. Therefore let $G=S L(2, \mathbb{R})$ be the complex Lie group, which is 


$$
S L(2, \mathbb{R})=\left\{\left(\begin{array}{ll}
a & b \\
c & d
\end{array}\right):(a, b, c, d) \in \mathbb{R}^{4} \text { and } a d-b c=1\right\}
$$

and let $G=K N A$ be the Iwasawa decomposition of $G$, where

$$
\begin{aligned}
& K=K(G)=\left\{\left(\begin{array}{cc}
\cos \phi & -\sin \phi \\
\sin \phi & \cos \phi
\end{array}\right)=S O(2): \phi \in \mathbb{R}\right\} \\
& N=N(G)=\left\{\left(\begin{array}{cc}
1 & n \\
0 & 1
\end{array}\right): n \in \mathbb{R}\right\} \\
& A=A(G)=\left\{\left(\begin{array}{cc}
a & 0 \\
0 & a^{-1}
\end{array}\right): a \in \mathbb{R}_{+}^{\star}\right\}
\end{aligned}
$$

Hence every $g \in G$ can be written as $g=k a n \in G$, where $k \in K, a \in A$, $n \in \mathbb{R}$. We denote by $L^{1}(G)$ the Banach algebra that consists of all complex valued functions on the group $G$, which are integrable with respect to the Haar measure $d g$ of $G$ and multiplication is defined by convolution product on $G$, and we denote by $L^{2}(G)$ the Hilbert space of $G$. So we have for any $f \in L^{1}(G)$ and $\phi \in L^{1}(G)$

$$
\phi * f(h)=\int_{G} f\left(g^{-1} h\right) \phi(g) d g
$$

The Haar measure $d g$ on a connected real semi-simple Lie group $G=S L(n, \mathbb{R})$, can be calculated from the Haar measures $d n$; $d a$ and $d k$ on $N ; A$ and $K$, respectively, by the formula

$$
\int_{G} f(g) d g=\int_{A} \int_{N} \int_{K} f(a n k) d a d n d k
$$

Keeping in mind that $a^{-2 \rho}$ is the modulus of the automorphism $n \rightarrow a n a^{-1}$ of $N$ we get also the following representation of $d g$

$$
\int_{G} f(g) d g=\int_{A} \int_{N} \int_{K} f(a n k) d a d n d k=\iint_{N} \int_{A} \int_{K} f(n a k) a^{-2 \rho} d n d a d k
$$

where

$$
\rho=2^{-1} \sum_{\alpha>0} m(\alpha) \alpha
$$


and $m(\alpha)$ denotes the multiplicity of the root $\alpha$ see [17] or again $\rho=$ the dimension of the nilpotent group $N$. Furthermore, using the relation $\int_{G} f(g) d g=$ $\int_{G} f\left(g^{-1}\right) d g$, we receive

$$
\int_{G} f(g) d g=\int_{K} \int_{A} \int_{N} f(k a n) a^{2 \rho} d n d a d k
$$

Let $\Gamma$ be a connected compact Lie group and let $\underline{k}$ be the Lie algebra of $\Gamma$. Let $\left(X_{1}, X_{2}, \ldots . ., X_{m}\right)$ a basis of $\underline{k}$, such that the both operators

$$
\begin{gathered}
\Delta=\sum_{i=1}^{m} X_{i}^{2} \\
D_{q}=\sum_{0 \leq l \leq q}\left(-\sum_{i=1}^{m} X_{i}^{2}\right)^{l}
\end{gathered}
$$

are left and right invariant (bi-invariant) on $\Gamma$, this basis exist see $[2, p .564$ ). For $l \in \mathbb{N}$, let $D^{l}=(1-\Delta)^{l}$, then the family of semi-norms $\left\{\sigma_{l}, l \in \mathbb{N}\right\}$ such that

$$
\left.\sigma_{l}(f)=\int_{\Gamma}\left|D^{l} f(y)\right|^{2} d y\right)^{\frac{1}{2}}, \quad f \in C^{\infty}(\Gamma)
$$

define on $C^{\infty}(\Gamma)$ the same topology of the Frechet topology defined by the semi-normas $\left\|X^{\alpha} f\right\|_{2}$ defined as

$$
\left\|X^{\alpha} f\right\|_{2}=\int_{\Gamma}\left(\left|X^{\alpha} f(y)\right|^{2} d y\right)^{\frac{1}{2}}, \quad f \in C^{\infty}(\Gamma)
$$

where $\alpha=\left(\alpha_{1}, \ldots ., \alpha_{m}\right) \in \mathbb{N}^{m}$, see $[2, p .565]$

Let $\widehat{\Gamma}$ be the set of all irreducible unitary representations of $\Gamma$. If $\gamma \in \widehat{\Gamma}$, we denote by $E_{\gamma}$ the space of representation $\gamma$ and $d_{\gamma}$ its dimension then we get

Definition 2.1. The Fourier transform of a function $f \in C^{\infty}(\Gamma)$ is defined as

$$
T f(\gamma)=\int_{\Gamma} f(x) \gamma\left(x^{-1}\right) d x
$$

where $T$ is the Fourier transform on $\Gamma$

Theorem (A. Cerezo) 2.1. Let $f \in C^{\infty}(\Gamma)$, then we have the inversion of the Fourier transform

$$
f(x)=\sum_{\gamma \in \widehat{\Gamma}} d \gamma \operatorname{tr}[T f(\gamma) \gamma(x)
$$




$$
f\left(I_{\Gamma}\right)=\sum_{\gamma \in \widehat{\Gamma}} d \gamma \operatorname{tr}[T f(\gamma)]
$$

and the Plancherel formula

$$
\|f(x)\|_{2}^{2}=\int_{\Gamma}|f(x)|^{2} d x=\sum_{\gamma \in \widehat{\Gamma}} d_{\gamma}\|T f(\gamma)\|_{H . S}^{2}
$$

for any $f \in L^{1}(\Gamma)$, where $I_{\Gamma}$ is the identity element of $\Gamma$ and $\|T f(\gamma)\|_{H . S}^{2}$ is the Hilbert-Schmidt norm of the operator $T f(\gamma)$

Fourier did not actually assume any underlying group structure or representation theory but we typically associate his work with the case of the circle group in the following form using complex exponentials

$$
f(x)=\sum_{n=-\infty}^{\infty} T f(m) e^{i x m}=\sum_{m=-\infty}^{\infty} c_{n} e^{i x m}, \quad m \in \mathbb{Z}
$$

where

$$
c_{m}=T f(m)=\int_{S O(2)} f(x) e^{-i x m} d x
$$

The group is $S O(2)=S^{1}$ or $\mathbb{R} / \mathbb{Z}$ and the multiplicative characters are $e^{i x n}$, group homomorphisms from the circle $K=S O(2)$ to the multiplicative group of non-zero complex numbers. Fourier actually preferred to express the coefficients using what is now known as the Plancherel formula

$$
\|f(x)\|_{2}^{2}=\int_{S O(2)}|f(x)|^{2} d x=\sum_{n=-\infty}^{\infty}\left|c_{m}\right|^{2}=\sum_{n=-\infty}^{\infty}|T f(m)|^{2}
$$

where

$$
S^{1}=S O(2)=\left\{\left(\begin{array}{cc}
\cos \phi & -\sin \phi \\
\sin \phi & \cos \phi
\end{array}\right): \phi \in \mathbb{R}\right\}
$$

Definition 2.2. For any function $f \in \mathcal{D}(G)$, we can define a function $\Upsilon(f)$ on $G \times K=G \times S O(2)$ by

$$
\Upsilon(f)\left(g, k_{1}\right)=\Upsilon(f)\left(k n a, k_{1}\right)=f\left(g k_{1}\right)=f\left(k n a k_{1}\right)
$$

for $g=k n a \in G$, and $k_{1} \in K$. The restriction of $\Upsilon(f) * \psi\left(g, k_{1}\right)$ on $K(G)$ is $\Upsilon(f) * \psi\left(g, k_{1}\right) \downarrow_{K(G)}=f\left(n a k_{1}\right)=f(g) \in \mathcal{D}(G)$, and $\Upsilon(f)\left(g, k_{1}\right) \downarrow_{K}=f(k n a)$ $\in \mathcal{D}(G)$ 
Remark 2.1. $\Upsilon(f)$ is invariant in the following sense

$$
\Upsilon(f)\left(g h, h^{-1} k_{1}\right)=\Upsilon(f)\left(g, k_{1}\right)
$$

Definition 2.3. If $f$ and $\psi$ are two functions belong to $\mathcal{D}(G)$, then we can define the convolution of $\Upsilon(f)$ and $\psi$ on $G \times K=G \times S^{1}=G \times S O(2)$ as

$$
\begin{aligned}
\Upsilon(f) * \psi\left(g, k_{1}\right) & =\int_{G} \Upsilon(f)\left(g g_{2}^{-1}, k_{1}\right) \psi\left(g_{2}\right) d g_{2} \\
& =\int_{S O(2)} \int_{N} \int_{A} \Upsilon(f)\left(k n a a_{2}^{-1} n_{2}^{-1} k^{-1} k_{1}\right) \psi\left(k_{2} n_{2} a_{2}\right) d k_{2} d n_{2} d a_{2}
\end{aligned}
$$

and so we get

$$
\begin{aligned}
\Upsilon(f) * \psi\left(g, k_{1}\right) & \downarrow K(G)=\Upsilon(f) * \psi\left(I_{K} n a, k_{1}\right) \\
& =\int_{S O(2)} \int_{N} \int_{A} f\left(n a a_{2}^{-1} n_{2}^{-1} k^{-1} k_{1}\right) \psi\left(k_{2} n_{2} a_{2}\right) d k_{2} d n_{2} d a_{2} \\
& =\Upsilon(f) * \psi\left(n a, k_{1}\right)
\end{aligned}
$$

where $g_{2}=k_{2} n_{2} a_{2}$

Definition 2.4. If $f \in \mathcal{D}(G)$ and let $\Upsilon(f)$ be the associated function to $f$ , we define the Fourier transform of $\Upsilon(f)\left(g, k_{1}\right)$ by

$$
\begin{aligned}
& \mathcal{F} \Upsilon(f))\left(I_{S^{1}}, \xi, \lambda, \gamma, I_{S^{1}}\right)=\mathcal{F} \Upsilon(f)\left(I_{S^{1}}, \xi, \lambda, I_{S^{1}}\right) \\
= & \int_{S^{1}} \int_{N} \int_{A}\left[\sum_{l=-\infty}^{\infty} \int_{S^{1}} T \Upsilon(f)\left(k n a, k_{1}\right) e^{-i l k} d k\right] a^{-i \lambda} e^{-i\langle\xi, n\rangle} e^{-i m k_{1}} d a d n d k_{1} \\
= & \int_{S^{1}} \int_{N} \int_{A}\left[\Upsilon(f)\left(I_{S^{1}} n a, k_{1}\right)\right] a^{-i \lambda} e^{-i\langle\xi, n\rangle} e^{-i m k_{1}} d a d n d k_{1}
\end{aligned}
$$

where $\mathcal{F}$ is the Fourier transform on $A N$ and $T$ is the Fourier transform on $S O(2)$, and $I_{S^{1}}$ is the identity element of $S^{1}=S O(2)$

Plancherel's Theorem on the Group G 2.2. For any function $f \in$ $L^{1}(G) \cap L^{2}(G)$, we get

$$
\int_{G}|f(g)|^{2} d g=\int_{A} \int_{N} \int_{S^{1}}|f(k n a)|^{2} d a d n d k=\sum_{m=-\infty}^{\infty} \int_{\mathbb{R}} \int_{\mathbb{R}}\|T \mathcal{F} f(\lambda, \xi, m)\|_{2}^{2} d \lambda d \xi
$$


$f\left(I_{A} I_{N} I_{S^{1}}\right)=\int_{N} \int_{A} \sum_{m=-\infty}^{\infty} T \mathcal{F} f((\lambda, \xi, m)] d \lambda d \xi=\sum_{m=-\infty}^{\infty} \int_{\mathbb{R}} \int_{\mathbb{R}} T \mathcal{F} f(\lambda, \xi, m) d \lambda d \xi$

where $I_{A}, I_{N}$, and $I_{K}$ are the identity elements of $A, N$ and $K$ respectively, where $\mathcal{F}$ is the Fourier transform on $A N$ and $T$ is the Fourier transform on $K$, and $I_{K}$ is the identity element of $K$

Proof: First let $\stackrel{\vee}{f}$ be the function defined by

$$
\stackrel{\vee}{f}(k n a)=\overline{f\left((k n a)^{-1}\right)}=\overline{f\left(a^{-1} n^{-1} k^{-1}\right)}
$$

Then we have

$$
\begin{aligned}
& \int_{G}|f(g)|^{2} d g \\
= & \Upsilon(f) * f\left(I_{S^{1}} I_{N} I_{A}, I_{S^{1}}\right) \\
= & \int_{G} \Upsilon(f)\left(I_{S^{1}} I_{N} I_{A}\left(g_{2}^{-1}\right), I_{S^{1}}\right) f^{\vee}\left(g_{2}\right) d g_{2} \\
= & \int_{A} \int_{N} \int_{S^{1}} \Upsilon(f)\left(a_{2}^{-1} n_{2}^{-1} k_{2}^{-1}, I_{S^{1}}\right) f^{\vee}\left(k_{2} n_{2} a_{2}\right) d a_{2} d n_{2} d k_{2} \\
= & \int_{A} \int_{N} \int_{S^{1}} f\left(a_{2}^{-1} n_{2}^{-1} k_{2}^{-1}\right) \overline{f\left(\left(k_{2} n_{2} a_{2}\right)^{-1}\right)} d a_{2} d n_{2} d k_{2} \\
= & \int_{A} \int_{N} \int_{S^{1}}\left|f\left(a_{2} n_{2} k_{2}\right)\right|^{2} d a_{2} d n_{2} d k_{2}
\end{aligned}
$$

Secondly

$$
\begin{aligned}
& \Upsilon(f) * f\left(I_{S^{1}} I_{N} I_{A}, I_{S^{1}}\right) \\
= & \int_{\mathbb{R}} \int_{\mathbb{R}} \mathcal{F}(\Upsilon(f) * f)\left(I_{S^{1}}, \lambda, \xi, I_{S^{1}}\right) d \lambda d \xi \\
= & \int_{S^{1}} \int_{\mathbb{R}} \int_{\mathbb{R}} \int_{A} \int_{N_{N}} \sum_{m=-\infty}^{\infty} \sum_{l=-\infty}^{\infty} \int_{S^{1}} \Upsilon(f) * f\left(k n a, k_{1}\right) e^{-i l k} d k a^{-i \lambda} \\
& e^{-i\langle\xi, n\rangle} e^{-i m k_{1}} d a d n d k_{1} d \lambda d \xi
\end{aligned}
$$




$$
\begin{aligned}
= & \sum_{m=-\infty}^{\infty} \int_{S^{1}} \int_{\mathbb{R}} \int_{\mathbb{R}} \int_{A} \int_{N} \Upsilon(f) * f\left(I_{S^{1}} n a, k_{1}\right) \\
& e^{-i l k} d k a^{-i \lambda} e^{-i\langle\xi, n\rangle} e^{-i m k_{1}} d a d n d k_{1} d \lambda d \xi \\
= & \int_{\mathbb{R}} \int_{\mathbb{R}} \int_{A} \int_{N} \int_{S^{1}} \int_{A} \int_{N} \sum_{m=-\infty}^{\infty} \int_{S^{1}} \Upsilon(f)\left(I_{S^{1}} n a a_{2}^{-1} n_{2}^{-1} k_{2}^{-1}, k_{1}\right) f\left(k_{2} n_{2} a_{2}\right) e^{-i m k_{1}} d k_{1} \\
& d n d a d k_{2} d n_{2} d a_{2} a^{-i \lambda} e^{-i\langle\xi, n\rangle} d \lambda d \xi
\end{aligned}
$$$$
=\int_{\mathbb{R}} \int_{\mathbb{R}} \int_{A} \int_{N} \int_{S^{1}} \int_{A} \int_{N} \sum_{m=-\infty}^{\infty} \int_{S^{1}} f\left(n a a_{2}^{-1} n_{2}^{-1} k_{2}^{-1} k_{1}\right) f\left(k_{2} n_{2} a_{2}\right) e^{-i m k_{1}} d k_{1} d k_{2}
$$$$
a^{-i \lambda} e^{-i\langle\xi, n\rangle} d n d a d n_{2} d a_{2} d \lambda d \xi
$$

where

$$
e^{-i\langle\xi, n\rangle}=e^{-i \xi n}
$$

Using the fact that

$$
\int_{A} \int_{N} \int_{S^{1}} f(k n a) d a d n d k=\int_{N} \int_{A} \int_{S^{1}} f(k a n) a^{2} d n d a d k
$$

and

$$
\begin{aligned}
& \int_{\mathbb{R}} \int_{A} \int_{N} \int_{S^{1}} f(k n a) e^{-i\langle\xi, n\rangle} d a d n d k d d \xi \\
= & \int_{\mathbb{R}} \int_{A} \int_{N} \int_{S^{1}} f(k a n) e^{-i\left\langle\xi, a n_{1} a^{-1}\right\rangle} a^{2} d a d n d k d \xi \\
= & \int_{\mathbb{R}} \int_{A} \int_{N} \int_{S^{1}} f(k a n) e^{-i\left\langle a \xi a^{-1}, n\right\rangle} a^{2} d a d n d k d \xi \\
= & \int_{\mathbb{R}} \int_{A} \int_{N} \int_{S^{1}} f(k a n) e^{-i\langle\xi, n\rangle} d a d n d k d \xi
\end{aligned}
$$

Then we get

$$
\begin{aligned}
& \Upsilon(f) * f\left(I_{S^{1}} I_{N} I_{A}, I_{S^{1}}\right) \\
= & \int_{\mathbb{R}} \int_{\mathbb{R}} \int_{A} \int_{N} \int_{S^{1}} \int_{A} \int_{N} \sum_{m=-\infty}^{\infty} \int_{S^{1}} f\left(n a a_{2}^{-1} n_{2}^{-1} k_{2}^{-1}, k_{1}\right) f\left(k_{2} n_{2} a_{2}\right) e^{-i m k_{1}} d k_{1} d k_{2}
\end{aligned}
$$




$$
\begin{aligned}
& a^{-i \lambda} e^{-i\langle\xi, n\rangle} d n d a d n_{2} d a_{2} d \lambda d \xi \\
& =\int_{\mathbb{R}} \int_{\mathbb{R}} \int_{A} \int_{N} \int_{A} \int_{N} \sum_{m=-\infty}^{\infty} \int_{S^{1}} \int_{S^{1}} f\left(a a_{2}^{-1} n n_{2}^{-1} k_{2}^{-1}, k_{1}\right) f\left(k_{2} n_{2} a_{2}\right) e^{-i m k_{1}} d k_{1} d k_{2} \\
& a^{-i \lambda} e^{-i\langle\xi, n\rangle} d n d a d n_{2} d a_{2} d \lambda d \xi \\
& =\int_{\mathbb{R}} \int_{\mathbb{R}} \int_{A} \int_{N} \int_{A} \int_{N} \sum_{m=-\infty}^{\infty} \int_{S^{1}} \int_{S^{1}} f\left(a n k_{2}^{-1}, k_{1}\right) f\left(k_{2} n_{2} a_{2}\right) e^{-i m k_{1}} d k_{1} d k_{2} \\
& a^{-i \lambda} e^{-i\langle\xi, n\rangle} d n d a d n_{2} d a_{2} d \lambda d \xi \\
& =\int_{\mathbb{R}} \int_{\mathbb{R}} \int_{A} \int_{N} \int_{A} \int_{N} \sum_{m=-\infty}^{\infty} \int_{S^{1}} \int_{S^{1}} f\left(a n k_{2}^{-1} k_{1}\right) f\left(k_{2} n_{2} a_{2}\right) e^{-i m k_{1}} d k_{1} d k_{2} \\
& a^{-i \lambda} e^{-i\langle\xi, n\rangle} d n d a d n_{2} d a_{2} d \lambda d \xi \\
& =\int_{\mathbb{R}} \int_{\mathbb{R}} \int_{A} \int_{N} \int_{A} \int_{N} \sum_{m=-\infty}^{\infty} \int_{S^{1}} \int_{S^{1}} f\left(a n k_{1}^{-1}\right) f\left(k_{2} n_{2} a_{2}\right) e^{-i m k_{1}} e^{-i m k_{2}} d k_{1} d k_{2} \\
& a^{-i \lambda} a_{2}^{-i \lambda} e^{-i\left\langle\xi, n+n_{2}\right\rangle} d n d a d n_{2} d a_{2} d \lambda d \xi \\
& =\int_{\mathbb{R}} \int_{\mathbb{R}} \int_{A} \int_{N} \int_{A} \int_{N} \sum_{m=-\infty}^{\infty} \int_{S^{1}} \int_{S^{1}} f\left(a n k_{1}^{-1}\right) \overline{f\left(a_{2}^{-1} n_{2}^{-1} k_{2}^{-1}\right)} e^{-i m k_{1}} e^{-i m k_{2}} d k_{1} d k_{2} \\
& a^{-i \lambda} e^{-i\langle\xi, n\rangle} a_{2}^{-i \lambda} e^{-i\left\langle\xi, n_{2}\right\rangle} d n d a d n_{2} d a_{2} d \lambda d \xi \\
& =\int_{\mathbb{R}} \int_{\mathbb{R}} \int_{A} \int_{N} \int_{A} \int_{N} \sum_{m=-\infty}^{\infty} \int_{S^{1}} \int_{S^{1}} f\left(a n k_{1}^{-1}\right) \overline{f\left(a_{2} n_{2} k_{2}\right) e^{-i m k_{2}}} e^{-i m k_{1}} d k_{1} d k_{2} \\
& a^{-i \lambda} e^{-i\langle\xi, n\rangle} a_{2}^{-i \lambda} e^{i\left\langle\xi, n_{2}\right\rangle} d n d a d n_{2} d a_{2} d \lambda d \xi \\
& =\int_{\mathbb{R}} \int_{\mathbb{R}} \int_{A} \int_{N} \int_{A} \int_{N} \sum_{m=-\infty}^{\infty} \int_{S^{1}} \int_{S^{1}} f\left(a n k_{1}^{-1}\right) \overline{f\left(a_{2} n_{2} k_{2}\right) e^{-i m k_{2}}} e^{-i m k_{1}} d k_{1} d k_{2} \\
& a^{-i \lambda} e^{-i\langle\xi, n\rangle} \overline{a_{2}^{-i \lambda} e^{-i\left\langle\xi, n_{2}\right\rangle}} d n d a d n_{2} d a_{2} d \lambda d \xi \\
& =\int_{\mathbb{R}} \int_{\mathbb{R}} \sum_{m=-\infty}^{\infty} \operatorname{TFf}(\lambda, \xi, m) \overline{\operatorname{TF} f(\lambda, \xi, m)} d \lambda d \xi \\
& =\int_{\mathbb{R}} \int_{\mathbb{R}} \sum_{m=-\infty}^{\infty}|T \mathcal{F}(f)(\lambda, \xi, m)|^{2} d \lambda d \xi
\end{aligned}
$$




\section{Fourier Transform and Plancherel Formula on Group $\mathbb{R}^{2} \rtimes S L(2, \mathbb{R})$}

3. Let $P=\mathbb{R}^{2} \rtimes_{\rho} G=\mathbb{R}^{2} \rtimes_{\rho} S L(2, \mathbb{R})$ be the 5 -dimensional affine group. To define the Fourier transform on $P$ we need the definition of Fourier transform on $\mathbb{R}^{2} \rtimes S L(2, \mathbb{R})$. As well known the group $G L_{+}(2, \mathbb{R})=S L(2, \mathbb{R}) \times \mathbb{R}_{+}^{*}$ is the direct product of two Lie group $S L(2, \mathbb{R})$ and $\mathbb{R}_{+}^{*}$, where $\mathbb{R}_{+}^{*}=\{x \in \mathbb{R}, x>0\}$. Let $(v, g)$ and $\left(v^{\prime}, g^{\prime}\right)$ be two elements belong $P$, then the multiplication of $(v, g)$ and $\left(v^{\prime}, g^{\prime}\right)$ is given by

$$
(v, g)\left(v^{\prime}, g^{\prime}\right)=\left(v+\rho(g)\left(v^{\prime}\right), g g^{\prime}\right)=\left(v+g v^{\prime}, g g^{\prime}\right)
$$

for any $\left(v, v^{\prime}\right) \in \mathbb{R}^{2} \times \mathbb{R}^{2}$ and $\left(g, g^{\prime}\right) \in G \times G$, where $g v^{\prime}=\rho(g)\left(v^{\prime}\right)$. To define the Fourier transform on $G$, we introduce the following new group

Definition 3.1. Let $Q=\mathbb{R}^{2} \times G \times G$ be the group with law:

$$
\begin{aligned}
X \cdot Y & =(v, h, g)\left(v^{\prime}, h^{\prime}, g^{\prime}\right) \\
& =\left(v+g v^{\prime}, h h^{\prime}, g g^{\prime}\right)
\end{aligned}
$$

for all $X=(v, h, g) \in Q$ and $Y=\left(v^{\prime}, h^{\prime}, g^{\prime}\right) \in Q$. Denote by $A=\mathbb{R}^{2} \times G$ the group of the direct product of $\mathbb{R}^{2}$ with the group $G$. then the group $A$ can be regarded as the subgroup $\mathbb{R}^{2} \times G \times\left\{I_{G}\right\}$ of $Q$ and $P$ can be regarded as the subgroup $\mathbb{R}^{2} \times\left\{I_{G}\right\} \times G$ of $Q$.

Definition 3.2. For any function $f \in \mathcal{D}(P)$, we can define a function $\tilde{f}$ on $Q$ by

$$
\widetilde{f}(v, g, h)=f(g v, g h)
$$

Remark 3.1. The function $\tilde{f}$ is invariant in the following sense

$$
\widetilde{f}\left(q^{-1} v, g, q^{-1} h\right)=\tilde{f}\left(v, g q^{-1}, h\right)
$$

Theorem 3.1. For any function $\psi \in \mathcal{D}(P)$ and $\tilde{f} \in C^{\infty}(Q)$ invariant in sense (32), we get

$$
\psi * \tilde{f}(v, h, g)=\widetilde{f} *_{c} \psi(v, h, g)
$$

where $*$ signifies the convolution product on $P$ with respect the variable $(v, g)$, and $*_{c}$ signifies the convolution product on $A$ with respect the variable $(v, h)$

Proof : In fact for each $\psi \in \mathcal{D}(P)$ and $\tilde{f} \in C^{\infty}(Q)$, we have

$$
\psi * \widetilde{f}(v, h, g)
$$




$$
\begin{aligned}
& =\int_{\mathbb{R}^{2}} \int_{G} \tilde{f}\left(\left(v^{\prime}, g^{\prime}\right)^{-1}(v, h, g)\right) \psi\left(v^{\prime}, g^{\prime}\right) d v^{\prime} d g^{\prime} \\
& =\int_{\mathbb{R}^{2}} \int_{G} \tilde{f}\left[\left(g^{\prime-1}\left(-v^{\prime}\right), g^{\prime-1}\right)(v, h, g)\right] \psi\left(v^{\prime}, g^{\prime}\right) d v^{\prime} d g^{\prime} \\
& =\int_{\mathbb{R}^{2}} \int_{G} \tilde{f}\left[\left(g^{\prime-1}\left(-v^{\prime}\right), g^{\prime-1}\right)(v, h, g)\right] \psi\left(v^{\prime}, g^{\prime}\right) d v^{\prime} d g^{\prime} \\
& =\int_{\mathbb{R}^{2}} \int_{G} \tilde{f}\left[\left(g^{\prime-1}\left(v-v^{\prime}\right), h, g^{\prime-1} g\right)\right] \psi\left(v^{\prime}, g^{\prime}\right) d v^{\prime} d g^{\prime} \\
& =\int_{\mathbb{R}^{2}} \int_{G} \tilde{f}\left[v-v^{\prime}, h g^{\prime-1}, g\right] \psi\left(v^{\prime}, g^{\prime}\right) d v^{\prime} d g^{\prime} \\
& =\widetilde{f} *_{c} \psi(v, h, g)
\end{aligned}
$$

Corollary 3.1. From theorem 3.1, the equation (35) turns as

$$
\begin{aligned}
& \psi * \widetilde{f}\left(v, h, I_{G}\right) \\
= & \psi *_{c} \widetilde{f}\left(v, h, I_{G}\right)=\int_{\mathbb{R}^{2}} \int_{G} \tilde{f}\left[v-v^{\prime}, h g^{\prime-1}, g\right] \psi\left(v^{\prime}, g^{\prime}\right) d v^{\prime} d g^{\prime} \\
= & \int_{\mathbb{R}^{2}} \int_{G} f\left[h g^{\prime-1}\left(v-v^{\prime}\right), h g^{\prime-1}\right] \psi\left(v^{\prime}, g^{\prime}\right) d v^{\prime} d g^{\prime}=h(f) *_{c} \psi(v, h)
\end{aligned}
$$

where

$$
h(f)(v, g)=f(g v, g)
$$

Definition 3.3. Let $\Upsilon F$ be the function on $P \times S^{1}$ defined by

$$
\Upsilon F\left(v,\left(g, k_{1}\right)\right)=F\left(v, g k_{1}\right)
$$

Definition 3.4. Let $\psi \in \mathcal{D}(P)$ and $F \in \mathcal{D}(P)$, then we can define a convolution product on the Affine group $P$ as

$$
\begin{aligned}
\psi *_{c} \Upsilon F\left(v,\left(g, k_{1}\right)\right) & =\int_{\mathbb{R}^{2}} \int_{G} \Upsilon F\left(v-v^{\prime},\left(g g^{\prime-1}, k_{1}\right)\right) \psi\left(v^{\prime}, g^{\prime}\right) d v^{\prime} d g^{\prime} \\
& \left.=\int_{\mathbb{R}^{2}} \int_{K} \int_{N} \int_{A} F\left(v-v^{\prime}, k n a\left(k^{\prime} n^{\prime} a^{\prime}\right)^{-1} k_{1}\right)\right)
\end{aligned}
$$




$$
\psi\left(v^{\prime}, k^{\prime} n^{\prime} a^{\prime}\right) d v^{\prime} d k^{\prime} d n^{\prime} d a^{\prime}
$$

where $g=k n a$ and $g^{\prime}=k^{\prime} n^{\prime} a^{\prime}$

Corollary 3.2. For any function $F$ belongs to $\mathcal{D}(P)$, we obtain

$$
\begin{aligned}
\psi *_{c} \Upsilon h(F)\left(v,\left(g, k_{1}\right)\right) & =\int_{\mathbb{R}^{2}} \int_{G} \Upsilon h(F)\left(v-v^{\prime},\left(g g^{\prime-1}, k_{1}\right) \psi\left(v^{\prime}, g^{\prime}\right) d v^{\prime} d g^{\prime}\right. \\
& =\int_{\mathbb{R}^{2}} \int_{G} \Upsilon h(F)\left(v-v^{\prime},\left(g g^{\prime-1}, k_{1}\right) \psi\left(v^{\prime}, g^{\prime}\right) d v^{\prime} d g^{\prime}\right. \\
& =\int_{\mathbb{R}^{2}} \int_{G} h(F)\left(v-v^{\prime}, g g^{\prime-1} k_{1}\right) \psi\left(v^{\prime}, g^{\prime}\right) d v^{\prime} d g^{\prime} \\
& =\int_{\mathbb{R}^{2}} \int_{G} F\left(g g^{\prime-1} k_{1}\left(v-v^{\prime}\right), g g^{\prime-1} k_{1}\right) \psi\left(v^{\prime}, g^{\prime}\right) d v^{\prime} d g^{\prime}
\end{aligned}
$$

Corollary 3.3. For any function $F$ belongs to $\mathcal{D}(P)$, we obtain

$$
F * \Upsilon h(\stackrel{\vee}{F})\left(0,\left(I_{G}, I_{S^{1}}\right)\right)=\int_{G} \int_{\mathbb{R}^{2}}|f(v, g)|^{2} d g d v=\|f\|_{2}^{2}
$$

Proof: If $F \in \mathcal{D}(P)$,then we get

$$
\begin{aligned}
& F * \Upsilon h(\stackrel{\vee}{F})\left(0,\left(I_{G}, I_{S^{1}}\right)\right) \\
& =\int_{G} \int_{\mathbb{R}^{2}} \Upsilon \hbar(\stackrel{\vee}{F})\left[(0-v),\left(I_{G} g^{-1}, I_{S^{1}}\right)\right] F(v, g) d g d v \\
& =\int_{G} \int_{\mathbb{R}^{2}} \hbar(\stackrel{\vee}{F})\left[(0-v), I_{G} g^{-1} I_{S^{1}}\right] F(v, g) d g d v \\
& =\int_{G} \int_{\mathbb{R}^{2}} \hbar(\stackrel{\vee}{F})\left[(-v), g^{-1}\right] F(v, g) d g d v \\
& =\int_{G} \int_{\mathbb{R}^{2}} \stackrel{\vee}{F}\left[g^{-1}(-v), g^{-1}\right] F(v, g) d g d v \\
& =\int_{G} \int_{\mathbb{R}^{2}} \overline{F\left[g^{-1}(-v), g^{-1}\right]^{-1}} F(v, g) d g d v=\int_{G} \int_{\mathbb{R}^{4}} \overline{F[v, g]} F(v, g) d g d v
\end{aligned}
$$




$$
=\int_{G} \int_{\mathbb{R}^{2}}|f(v, g)|^{2} d g d v
$$

Definition 3.5. Let $f \in \mathcal{D}(P)$, we define its Fourier transform by

$$
\begin{aligned}
\mathcal{F}_{\mathbb{R}^{2}} & \operatorname{TFf}(\eta, m, \xi, \lambda) \\
& =\int_{\mathbb{R}^{2}} \int_{A} \int_{N} \int_{K} f(v, k n a) e^{-i\langle\eta, v\rangle} e^{-i m k} a^{-i \lambda} e^{-i\langle\xi, n\rangle} d k d a d n d \lambda d \xi d v
\end{aligned}
$$

where $\mathcal{F}_{\mathbb{R}^{2}}$ is the Fourier transform on $\mathbb{R}^{2}$, kna $=g, \eta=\left(\eta_{1}, \eta_{2}\right) \in \mathbb{R}^{2}, v=$ $\left(v_{1}, v_{2}\right) \in \mathbb{R}^{2}$, and $d v=d v_{1} d v_{2}$ is the Lebesgue measure on $\mathbb{R}^{2}$ and

$$
\begin{aligned}
\langle\eta, v\rangle & =\left\langle\left(\eta_{1}, \eta_{2}\right),\left(v_{1}, v_{2}\right)\right\rangle \\
& =\eta_{1} v_{1}+\eta_{2} v_{2}
\end{aligned}
$$

To obtain the Plancherel formula for the $P$, we refer to $[7,8]$

Plancherel's Theorem 3.2. For any function $f \in L^{1}(P) \cap L^{2}(P)$, we get

$$
\int_{P}|f(v, g)|^{2} d v d g=\int_{\mathbb{R}} \int_{\mathbb{R}} \int_{\mathbb{R}} \sum_{m=-\infty}^{\infty}\left\|\mathcal{F}_{\mathbb{R}^{2}} T \mathcal{F} F(\eta, m, \xi, \lambda)\right\|^{2} d \eta d \lambda d \xi
$$

Proof: Let $\Upsilon \hbar(\stackrel{\vee}{F})$ be the function defined as

$$
\begin{aligned}
& \Upsilon \hbar(\stackrel{\vee}{F})\left(v ;\left(g, k_{1}\right)\right)=\hbar(\stackrel{\vee}{F})\left(v ; g k_{1}\right) \\
= & \stackrel{\vee}{F}\left(g k_{1} v ; g k_{1}\right)=\overline{\left.F\left(g k_{1} v ; g k_{1}\right)^{-1}\right)}
\end{aligned}
$$

then, we have

$$
\begin{aligned}
& F * \Upsilon \hbar(\stackrel{\vee}{F})\left(0,\left(I_{G}, I_{S^{1}}\right)\right)=F * \Upsilon \hbar(\stackrel{\vee}{F})\left(0,\left(I_{S^{1}} I_{N} I_{A}, I_{S^{1}}\right)\right) \\
= & \int_{\mathrm{R}} \int_{\mathrm{R}_{\mathrm{R}^{2}}} \int_{\mathrm{R}^{2}} \mathcal{F}_{\mathrm{R}^{2}} \mathcal{F}\left(F * \Upsilon \hbar(\stackrel{\vee}{F})\left(\eta,\left(I_{S^{1}}, \xi, \lambda, I_{S^{1}}\right)\right) d \lambda d \xi d \eta\right. \\
= & \iint_{\mathrm{R}} \int_{\mathrm{R}^{2}} \int_{\mathrm{R}} \int_{\mathrm{R}_{\mathrm{R}}} \int_{\mathrm{R}^{2}} \sum_{m=-\infty}^{\infty} \int_{S^{1}} \mathcal{F}_{\mathrm{R}^{2}} T \mathcal{F}(F * \Upsilon \hbar(\stackrel{\vee}{F}))\left(\left(\eta,\left(I_{S^{1}} n a, k_{1}\right)\right) e^{-i m k_{1}} d k_{1}\right) \\
& e^{-i\langle\eta, v\rangle} a^{-i \lambda} e^{-i\langle\xi, n\rangle} d a d n d v d \lambda d \xi d \eta \\
= & \int_{G} \int_{\mathrm{R}} \int_{\mathrm{R}} \int_{\mathrm{R}} \int_{\mathrm{R}_{\mathrm{R}^{2}}} \int_{\mathrm{R}^{2}} \int_{\mathrm{R}^{2}} \sum_{m=-\infty}^{\infty} \int_{S^{1}} \Upsilon \hbar(\stackrel{\vee}{F})\left((v-w),\left(I_{S^{1}} n a g_{2}^{-1}, k_{1}\right)\right) e^{-i m k_{1}} d k_{1} F\left(w, g_{2}\right) d g_{2}
\end{aligned}
$$




$$
\begin{aligned}
& e^{-i\langle\eta, v\rangle} a^{-i \lambda} e^{-i\langle\xi, n\rangle} d a d n d v d w d \lambda d \xi d \eta \\
& =\int_{\mathrm{R}} \int_{\mathrm{R}} \int_{\mathrm{R}} \int_{\mathrm{R}} \int_{\mathrm{R}} \int_{\mathrm{R}} \int_{\mathrm{R}^{2}} \int_{\mathrm{R}^{2}} \int_{\mathrm{R}^{2}} \int_{S^{1}} \sum_{m=-\infty}^{\infty} \int_{S^{1}} \hbar(\stackrel{\vee}{F})\left((v-w),\left(n a a_{2}^{-1} n_{2}^{-1} k_{2}^{-1} k_{1}\right)\right) e^{-i m k_{1}} d k_{1} \\
& F\left(w, k_{2} n_{2} a_{2}\right) d k_{2} e^{-i\langle\eta, v\rangle} a^{-i \lambda} e^{-i\langle\xi, n\rangle} d a_{2} d n_{2} d a d n d w d v d \lambda d \xi d \eta \\
& =\int_{\mathrm{R}} \int_{\mathrm{R}} \int_{\mathrm{R}} \int_{\mathrm{R}} \int_{\mathrm{R}} \int_{\mathrm{R}^{2}} \int_{\mathrm{R}^{2}} \int_{\mathrm{R}^{2}} \int_{\mathrm{R}^{2}} \int_{S^{1}} \sum_{m=-\infty}^{\infty} \int_{S^{1}} \hbar(\stackrel{\vee}{F})\left(v,\left(a n k_{1}\right)\right) e^{-i m k_{1}} d k_{1} F\left(w, k_{2} n_{2} a_{2}\right) e^{-i m k_{2}} d k_{2} \\
& e^{-i\langle\eta, v+w\rangle} a^{-i \lambda} a_{2}^{-i \lambda} e^{-i\left\langle\xi, n+n_{2}\right\rangle} d a_{2} d n_{2} d a d n d w d v d \lambda d \xi d \eta \\
& =\int_{\mathrm{R}} \int_{\mathrm{R}} \int_{\mathrm{R}} \int_{\mathrm{R}} \int_{\mathrm{R}} \int_{\mathrm{R}^{2}} \int_{\mathrm{R}^{2}} \int_{\mathrm{R}^{2}} \int_{\mathrm{R}^{2}} \int_{S^{1}} \sum_{m=-\infty}^{\infty} \int_{S^{1}}(\stackrel{\vee}{F})\left(a n k_{1} v, a n k_{1}\right) e^{-i m k_{1}} d k_{1} F\left(w, k_{2} n_{2} a_{2}\right) e^{-i m k_{2}} d k_{2} \\
& e^{-i\langle\eta, v\rangle} e^{-i\langle\eta, w\rangle} a^{-i \lambda} a_{2}^{-i \lambda} e^{-i\langle\xi, n\rangle} e^{-i\left\langle\xi, n_{2}\right\rangle} d a_{2} d n_{2} d a d n d w d v d \lambda d \xi d \eta \\
& =\int_{\mathrm{R}} \int_{\mathrm{R}} \int_{\mathrm{R}} \int_{\mathrm{R}} \int_{\mathrm{R}} \int_{\mathrm{R}} \int_{\mathrm{R}^{2}} \int_{\mathrm{R}^{2}} \int_{\mathrm{R}^{2}} \int_{S^{1}} \sum_{m=-\infty}^{\infty} \int_{S^{1}} \overline{F\left(-v, k_{1}^{-1} n^{-1} a^{-1}\right)} F\left(w, k_{2} n_{2} a_{2}\right) e^{-i m k_{1}} e^{-i m k_{2}} d k_{1} d k_{2} \\
& e^{-i\langle\eta, v\rangle} e^{-i\langle\eta, w\rangle} a^{-i \lambda} a_{2}^{-i \lambda} e^{-i\langle\xi, n\rangle} e^{-i\left\langle\xi, n_{2}\right\rangle} d a_{2} d n_{2} d a d n d w d v d \lambda d \xi d \eta \\
& =\int_{\mathrm{R}} \int_{\mathrm{R}} \int_{\mathrm{R}} \int_{\mathrm{R}} \int_{\mathrm{R}} \int_{\mathrm{R}} \int_{\mathrm{R}^{2}} \int_{\mathrm{R}^{2}} \int_{\mathrm{R}^{2}} \int_{S^{1}} \sum_{m=-\infty}^{\infty} \int_{S^{1}} \overline{F\left(v, k_{1} n a\right) e^{i m k_{1}}} F\left(w, k_{2} n_{2} a_{2}\right) e^{-i m k_{2}} d k_{1} d k_{2} \\
& e^{i\langle\eta, v\rangle} e^{-i\langle\eta, w\rangle} a^{i \lambda} a_{2}^{-i \lambda} e^{i\langle\xi, n\rangle} e^{-i\left\langle\xi, n_{2}\right\rangle} d a_{2} d n_{2} d a d n d w d v d \lambda d \xi d \eta \\
& \int_{\mathrm{R}} \int_{\mathrm{R}} \int_{\mathrm{R}^{2}} \sum_{m=-\infty}^{\infty}\left\|\mathcal{F}_{\mathrm{R}^{4}} T \mathcal{F} F(\eta, m, \xi, \lambda)\right\|_{H . S}^{2} d \eta d \lambda d \xi
\end{aligned}
$$

where $(0,0)$ is the identity element of the vector group $\mathbb{R}^{2}$ and $I_{S^{1}} I_{N} I_{A}$ is the identity element of the real semisimple Lie group $S L(2 ; \mathbb{R})=K N A$. Let $A F^{+}=\mathbb{R}^{2} \rtimes G L_{+}(2, \mathbb{R})$ be the group, which is the semidirect product of the group $G L_{+}(2, \mathbb{R})$ with the group $\mathbb{R}^{2}$. Since $G L_{+}(2, \mathbb{R})=S L(2 ; \mathbb{R}) \times \mathbb{R}_{+}^{*}$, then we get the following result

Theorem 3.3. For any function $F \in L^{1}\left(A F^{+}\right) \cap L^{2}\left(A F^{+}\right)$, we get

$$
\begin{aligned}
& \int_{A F^{+}}|F(v, g, a)|^{2} d v d g \frac{d a}{a} \\
&=\int_{\mathbb{R}} \int_{\mathbb{R}^{2}} \int_{\mathbb{R}} \int_{\mathbb{R}} \sum_{m=-\infty}^{\infty}\left\|\mathcal{F}_{\mathbb{R}^{2}} T \mathcal{F} F(\eta, m, \xi, \lambda, \mu)\right\|_{H . S}^{2} d \lambda d \xi d \eta d \mu
\end{aligned}
$$

As in my paper [7], we have proved that $G L_{-}(2, \mathbb{R})$ has structure of group which is isomorphic onto the group $G L_{+}(2, \mathbb{R})$, so we obtain

$$
G L(2, \mathbb{R})=G L_{+}(2, \mathbb{R}) \cup G L_{+}(2, \mathbb{R})
$$

Now our final state result is 
Corollary 3.4. For any function $F \in L^{1}(A F) \cap L^{2}(A F)$, we get

$$
\begin{aligned}
& \int_{A F}|F(v, g, a)|^{2} d v d g \frac{d a}{a} \\
= & 2 \int_{A F}|F(v, g, a)|^{2} d v d g \frac{d a}{a} \\
= & 2 \int_{\mathbb{R}_{\mathbb{R}^{2}}} \int_{\mathbb{R}} \int_{\mathbb{R}} \sum_{m=-\infty}^{\infty}\left\|\mathcal{F}_{\mathbb{R}^{2}} T \mathcal{F} F(\eta, m, \xi, \lambda, \mu)\right\|_{H . S}^{2} d \lambda d \xi d \eta d \mu
\end{aligned}
$$

\section{References}

[1] G. S. Chirikjian., and A. Kyatkin, A., (2000), Engineering Applications in Non-commutative Harmonic Analysis, Johns Hopkins University, Baltimore, Maryland, CRC Press.

[2] A. Cerezo and F. Rouviere, "Solution elemetaire d'un operator differentielle invariant agauch sur un group de Lie reel compact" Annales Scientiques de E.N.S. 4 serie, tome 2, no 4,p 561-581, 1969.

[3] Einstein, Albert, (1926), "Space-Time", Encyclopedia Britannica, 13th ed.

[4] K. El- Hussein., (2009), Eigendistributions for the Invariant Differential operators on the Affine Group. Int. Journal of Math. Analysis, Vol. 3, no. 9, 419-429.

[5] K. El- Hussein., (2010), Fourier transform and invariant differential operators on the solvable Lie group G4, in Int. J. Contemp. Maths Sci. 5. No. 5-8, 403-417.

[6] K. El- Hussein., (2011), On the left ideals of group algebra on the affine group, Int. Math Forum, Int, Math. Forum 6, No. 1-4, 193-202.

[7] K. El- Hussein., (2013), Non Commutative Fourier Transform on Some Lie Groups and Its Application to Harmonic Analysis, International Journal of Engineering Research \& Technology (IJERT) Vol. 2 Issue 10, 2429- 2442.

[8] K. El- Hussein., (2013), Abstract Harmonic Analysis and Ideals of Banach Algebra on 3-Step Nilpotent Lie Groups. International Journal of Engineering Research \& Technology (IJERT), Vol. 2 Issue 11, NovemberVol. 2 Issue 11, November 
[9] Harish-Chandra; (1952), Plancherel formula for $2 \times 2$ real unimodular group, Proc. nat. Acad. Sci. U.S.A., vol. 38, pp. 337-342.

[10] Harish-Chandra; (1952), The Plancherel formula for complex semi-simple Lie group, Trans. Amer. Mth. Soc., vol. 76, pp. 485- 528.

[11] S. Helgason., (2005), The Abel, Fourier and Radon Transforms on Symmetric Spaces. Indagationes Mathematicae. 16, 531-551.

[12] A. A. Kirillov, ed, (1994), Representation Theory and Noncommutative Harmonic Analysis I, Springer- Verlag, Berlin.

[13] Matolcsi., (1994). Spacetime Without Reference Frames. Budapest: Akadémiai Kiad.

[14] Petkov, Vesselin., (2010). Minkowski Spacetime: A Hundred Years Later. Springer. p. 70. ISBN 90-481-3474-9., Section 3.4, p. 70.

[15] W.Rudin., (1962), Fourier Analysis on Groups, Interscience Publishers, New York, NY.

[16] A. Vaen Deal., (2007), The Fourier transform in quantum group theory, preprint (math.RA/0609502 at http://lanl.arXiv.org).

[17] G. Warner., (1970), Harmonic Analysis on Semi-Simple Lie Groups II, Springer-verlag Berlin Heidel;berg New york. 